\title{
Condutas terapêuticas para tratamento da Síndrome da Apnéia e Hipopnéia Obstrutiva do Sono (SAHOS) e da Síndrome da Resistência das Vias Aéreas Superiores (SRVAS) com enfoque no Aparelho Anti-Ronco (AAR-ITO)
}

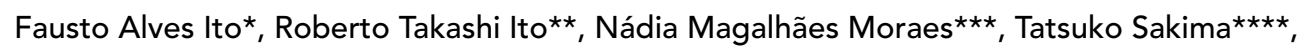

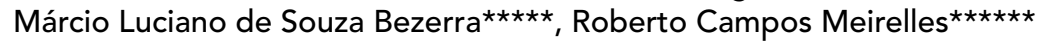

Resumo

Os autores apresentam uma revisão da literatura referente às alternativas de tratamento cirúrgicas e não-cirúrgicas para a Síndrome da Apnéia e Hipopnéia Obstrutiva do Sono (SAHOS) e para a Síndrome da Resistência das Vias Aéreas superiores (SRVAS) com enfoque no princípio de ação e evolução dos aparelhos de avanço mandibular e, em particular, no mecanismo dinâmico de ação do Aparelho Anti-Ronco® (AAR-ITO).

Palavras-chave: Aparelho Anti-Ronco $®$ (AAR-ITO). Aparelhos de avanço mandibular. Mecanismo dinâmico de ação. Síndrome da Resistência das Vias Aéreas superiores (SRVAS). Síndrome da Apnéia e Hipopnéia Obstrutiva do Sono (SAHOS).

\section{INTRODUÇÃO}

O fenômeno sono é essencial à homeostasia e por meio do qual todo o organismo, incluindo o sistema nervoso central, pode ser renovado. É definido como processo dinâmico e fisiológico de perda de consciência e inativação da musculatura voluntária, sendo reversível frente a estímulos (tátil, auditivo, somato-sensitivo). Diversas teorias foram propostas e pode-se afirmar que as funções do sono sofreram mudanças de acordo com a evolução dos mamíferos. Iniciou-se, principalmente, como um modo de tornar o animal imóvel e assim conservar energia e culminou com os humanos como um meio de facilitar o descanso e recuperar as funções cerebrais. Apresenta variações quanto à distribuição dos estágios e ao ritmo circadiano conforme a faixa etária, podendo ser mais fragmentado na população idosa, que além disso, tem maior tendência aos cochilos diurnos ${ }^{7,47,58}$.

A estrutura normal do sono é composta de 5 fases: estágios 1, 2, 3, 4 do sono lento ou NREM (Non Rapid Eyes Moviment $=$ sem movimentos oculares rápidos) e sono paradoxal ou REM (Rapid eyes moviment $=$ movimentos oculares rápidos).

* Cirurgião-Dentista (UNESA-RJ), Especialista em Anatomia Aplicada da Cabeça (ICB-USP), Aluno do Curso de Mestrado da Faculdade de Ciências Médicas da Universidade do Estado do Rio de Janeiro (UERJ).

** Cirurgião-Dentista (UFF-RJ).

*** Professora Adjunta do Departamento de Odontologia Social e Preventiva da Universidade Federal do Rio de Janeiro (UFRJ).

**** Professor Titular do Departamento de Ortodontia da Faculdade de Odontologia de Araraquara - São Paulo (UNESP). $\star \star \star \star \star$ Diretor do Instituto do Sono da Universidade Estácio de Sá (UNESA-RJ).

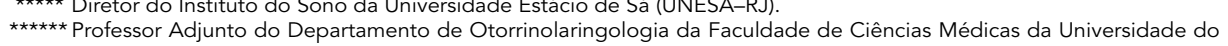
Estado do Rio de Janeiro (UERJ) 
Estes estágios são cíclicos e apresentam alterações fisiológicas e comportamentais específicas nos quais o sono e a vigília se alternam no tempo durante a noite. Normalmente, em um adulto que dorme cerca de 7 a 8 horas, ocorrem cerca de 4 a 6 ciclos por noite, sendo necessários cerca de 70 a 120 minutos para completar cada ciclo. Entretanto, alguns indivíduos necessitam de um número menor de horas de sono (dormidores curtos) e outros necessitam de mais horas (dormidores longos) para se restabelecerem física e mentalmente ${ }^{2,57,58}$.

A Síndrome da Resistência das Vias Aéreas superiores (SRVAS) e a Síndrome da Apnéia e Hipopnéia Obstrutiva do Sono (SAHOS) são consideradas transtornos respiratórios do sono que afetam a população geral com capacidade de fragmentar a arquitetura do sono, devido aos recorrentes microdespertares noturnos e às pausas respiratórias que, por conseqüência, poderão acarretar alterações funcionais, neurocognitivas e psicossociais $^{14,20,41,44}$.

A classificação internacional dos distúrbios do sono, publicada em 1997, definiu a SAHOS como dissônia, ou seja, transtorno que leva à hipersonolência diurna ou dificuldade de iniciar ou manter o sono.

\section{PROPOSIÇÃO}

O objetivo desta revisão de literatura é descrever as estratégias de tratamento para a SAHOS e para a SRVAS.

\section{EPIDEMIOLOGIA}

Dados epidemiológicos revelam que a SRVAS e a SAHOS apresentam alta incidência e prevalência, atingindo $2 \%$ da população feminina e $4 \%$ da masculina. Podem acometer qualquer faixa etária, entretanto, o pico de incidência está situado entre 40 e 60 anos. A SAHOS é considerada problema de saúde pública, devido às conseqüências cardiovasculares e aos riscos de acidentes ocupacionais e automobilísticos em decorrência da hipersonolência diurna que se estabelece e das decisões equivocadas que ocorrem. Está relacionada com a má qualidade de vida do paciente acometido, com deterioração dos aspectos neurocognitivos, refletindo negativamente no relacionamento familiar, social e no rendimento intelectual e profissional $1^{4,6,20,29,53,56,69,73}$.

Dentre as comorbidades mais freqüentes da SAHOS estão as arritmias cardíacas, a hipertensão arterial sistêmica, infarto do miocárdio e acidente vascular cerebral ${ }^{11,15,43,64}$.

A faixa etária, condição hormonal e o gênero são fatores relevantes nessa síndrome, uma vez que estas variáveis podem estar relacionadas com diversas causas, sintomas e conseqüências, devido à complexidade dos mecanismos neuronais que envolvem o fenômeno sono e a patogênese da SAHOS $^{4,39}$.

\section{FISIOPATOLOGIA}

A obstrução das vias aéreas superiores durante o sono ocorre devido ao estreitamento dessas vias, que se estende desde a nasofaringe até a porção inferior da hipofaringe. A interação entre fatores fisiológicos e alterações anatômicas dessa região, são fundamentais para compreender a patogênese da SAHOS. Inúmeras causas têm sido sugeridas para elucidar a fisiopatologia desse transtorno, como: alterações nas estruturas craniofaciais; alterações no comando ventilatório; glossoptose; obesidade (aumento de tecido adiposo no tronco superior e na região cervical); volume aumentado de secreções respiratórias, hipertrofia de amígdalas e/ou adenóides, fatores extrínsecos tais como álcool, tabaco e drogas que deprimem o sistema nervoso central, além de componentes genéti$\cos ^{4,21,31,37,45,71}$.

Durante a vigília, o comando ventilatório é automático e voluntário ao passo que durante o sono esse comando passa a ser somente automático. Essa situação acarreta redução do controle ventilatório e, por conseqüência, poderá diminuir a modulação dos músculos dilatadores da faringe. Soma-se a isso, a posição de decúbito dorsal e a 
ação da gravidade que favorecem a pitose do músculo genioglosso em direção à parede posterior do conduto faríngeo, caracterizando o colapso na orofaringe $e^{4,6,27,37}$.

Em indivíduos com predisposição para a SAHOS, a pressão intraluminal negativa produzida pela contração diafragmática, em detrimento do esforço inspiratório, agrava a obstrução, evoluindo para apnéia. Em decorrência do quadro apnéico, se desenvolve hipoxemia e hipercapnia. Esses, estimulam o sistema nervoso central, que, por meio dos quimiorreceptores e mecanorreceptores, irão ativar núcleos da formação reticular do tronco cerebral levando ao despertar e por conseguinte, acionarão os músculos abdutores da faringe desobstruindo a mesma e permitindo o retorno do fluxo aéreo. Quando o paciente volta a dormir, os músculos relaxam e todo o processo se inicia novamente repetindo-se por várias vezes ao longo da noite , $^{41,27,40,41,71}$.

\section{QUADRO CLÍNICO}

As manifestações clínicas da SAHOS mais freqüentes e suas conseqüências são descritas a seguir: 1) sono inquieto, 2) despertares freqüentes (fragmentação do sono), 3) ronco, 4) apnéias, 5) déficits neurocognitivos (memória, atenção, concentração, reflexo), 6) hipersonolência diurna, 7) cefaléia matinal, 8) alterações comportamentais (mudança de humor, irritabilidade, fadiga crônica), 9) hipertensão arterial sistêmica (HAS) e hipertensão pulmonar (HP), 10) problemas sexuais (impotência), 11) policitemia, 12) estresse oxidativo e 13) dilatação da raiz da aorta ${ }^{20,27,64,69}$.

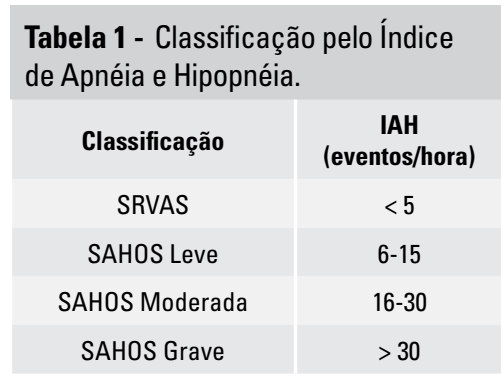

Fonte: American Sleep Disorders Association (1995).

\section{DIAGNÓSTICO}

Após avaliação clínica, é necessária a realização de um exame quantitativo específico, a polissonografia (PSG), que consiste no monitoramento de parâmetros fisiológicos durante o sono para que seja diagnosticado a gravidade, o tipo de apnéia presente, bem como alterações cardíacas, respiratórias e cerebrais. O histórico de ronco alto com hipersonolência diurna, associado ao excesso de peso, são algumas das principais justificativas para solicitação de um estudo polissonográfico, cuja acurácia e interpretação é de responsabilidade médica $^{6,16,22,26,27,40}$.

O critério diagnóstico para SAHOS refere-se à presença de cinco ou mais episódios de apnéia/ hipopnéia (IAH) por hora de sono acompanhado de diminuição da saturação de oxigênio sangüíneo menor que $85 \%$ (Tab. 1). O diagnóstico pode ser complementado por meio de análise cefalométrica, fibroscopia, tomografia computadorizada e ressonância nuclear magnética ${ }^{1}$.

\section{ESTRATÉGIAS DE TRATAMENTO}

Uma vez diagnosticado o transtorno, pode-se intervir com segurança no tratamento com o objetivo de devolver o bem-estar e a qualidade de vida ao paciente. Os mecanismos fisiopatológicos subjacentes à obstrução das vias aéreas superiores durante o sono, formam o alicerce para o tratamento da SRVAS e SAHOS $6,34,44$.

Sumariamente, as modalidades de tratamento buscam: 1) elevar a pressão na faringe acima da pressão obstrutiva; 2) reduzir a pressão obstrutiva; ou 3) aumentar a atividade muscular das vias aéreas superiores para ampliar o conduto faríngeo e permitir uma ventilação adequada durante o sono $^{40}$.

As medidas terapêuticas a serem tomadas irão depender da gravidade do transtorno diagnosticado, podendo variar desde: 1) medidas comportamentais, 2) utilização da máscara nasal - CPAP (Continuous Positive Airway Pressure $=$ Pressão Positiva e Contínua nas Vias Aéreas superiores), 
3) utilização de Aparelhos Intrabucais (AIOs), 4) tratamento farmacológico, 5) cirurgias e 6) terapias combinadas, como descrito a seguir ${ }^{9,21,26,37,54}$.

\section{1) Medidas comportamentais}

\section{1) Posição corporal}

Alguns estudos ${ }^{45,52,72}$ constataram que alterações no posicionamento do corpo têm influência sobre a estrutura das vias aéreas superiores. De acordo com dados polissonográficos, pacientes que dormem em decúbito dorsal apresentam roncos mais intensos e piora no quadro de apnéia por hora de sono. Desta forma, é recomendado ao paciente dormir na posição de decúbito lateral. Podese ainda orientar o mesmo a manter a cabeceira da cama inclinada cerca de 30 graus $4,13,26,37,45,60,67$.

\section{2) Relação entre obesidade e a SAHOS}

A prevalência da obesidade vem se tornando o maior problema de saúde da sociedade moderna. Em obesos grau 3 (mórbidos), com índice de massa corporal (IMC) igual ou superior a $40 \mathrm{~kg} / \mathrm{m}^{2}$, a incidência de SAHOS chega a ser cerca de 12 a 30 vezes maior quando comparada a pacientes com peso normal. Essas condições aumentam o risco de desenvolver diabetes mellitus (que está relacionada com xerostomia e infecções), dislipidemia e hipertensão arterial sistêmica, favorecendo o desenvolvimento de doenças cardiovasculares. A redução de peso é o tratamento ideal para esses pacientes, embora a taxa de sucesso a longo prazo seja considerada pequena quando avaliamos a cooperação dos mesmos. É lícito afirmar que a redução de peso, isoladamente, possui apenas efeito parcial, principalmente quando consideramos a gravidade da apnéia ${ }^{10,44,46,55,66,71}$.

\section{3) Álcool, sedativos e tabagismo}

As bebidas alcoólicas e drogas miorrelaxantes devem ser melhor avaliadas, pois reduzem o reflexo de despertar que ocorre durante os eventos obstrutivos, devido à depressão que provocam no sistema nervoso central. Essas substâncias, além de induzirem sono de má qualidade (não reparador), aumentam a freqüência e a duração das apnéias em pacientes sindrômicos (principalmente quando em decúbito dorsal), além de suscitar predisposição ao desenvolvimento de dependência química. Em adição, deve-se estimular também a descontinuação do uso de tabaco, devido à possibilidade de provocar edema e disfunção das vias aéreas superiores, o que aumenta a resistência ao fluxo aéreo $0^{4,19,36,37,63}$.

\section{4) Higiene do sono}

A higiene do sono consiste de algumas atitudes e medidas práticas saudáveis que visam a promoção de um sono contínuo e eficiente e que seja capaz de evitar a sonolência diurna no dia seguinte. A seguir descreveremos algumas orientações que devem ser passadas ao paciente de forma verbal e escrita para modificar hábitos inadequados com relação ao sono ${ }^{57}$ :

a) regularidade na hora de deitar e de despertar;

b) adequação do tempo em que se permanece na cama em relação ao tempo necessário para manter o sono adequado para cada pessoa;

c) exercícios físicos devem ser evitados próximo ao horário de dormir, porém mantidos regularmente todos os dias;

d) alimentação leve com intervalo mínimo de 2 horas antes de se deitar;

e) evitar o uso de substâncias que contenham cafeína e/ou nicotina por no mínimo 4 horas antes de dormir;

f) limitar ao mínimo o consumo de bebidas alcoólicas;

g) adequar o ambiente para favorecer o sono repousante, eliminando atividades incompatíveis como TV, lanches e telefone.

\section{2) Método físico-mecânico (CPAP)}

Existem alguns tipos de aparelhos de compressão de ar, tais como o CPAP, que foi adaptado e introduzido por Sullivan ${ }^{65}$, para tratar a SAHOS. Caracteriza-se por um método físico-mecânico de 
injeção de ar comprimido, utilizando máscara nasal, que tem por princípio manter a pressão positiva e contínua nas vias aéreas, desobstruindo a passagem de ar durante o sono. Apesar de eficiente, o CPAP apresenta baixa adesão a longo prazo $40,44,59,65$.

\section{3) Aparelhos intrabucais}

Historicamente, os dispositivos intrabucais utilizados para tratar a SAHOS e SRVAS são derivados dos aparelhos funcionais. Podemos descrevêlos e classificá-los como: 1) Aparelhos Retentores de Língua (ARL) (Fig. 1): são dispositivos confeccionados com material flexível que tracionam a língua por sucção mantendo-a anteriorizada por meio de bulbo localizado na região dos incisivos superiores e inferiores. São indicados para pacientes edêntulos totais; 2) Aparelhos Elevadores de Palato (AEP) (Fig. 2): esses possuem hastes metálicas para posterior que sustentam botão de acrílico em sua extremidade para elevar o palato mole e impedir a queda da úvula em direção à orofaringe. Estão em desuso devido ao desconforto e ao reflexo de vômito que provocam e 3) Aparelhos de Avanço Mandibular (AAM): estes dispositivos são os mais utilizados e investigados na literatura médica e odontológica. São indicados para pacientes dentados com quantidade de elementos dentários suficientes para ancoragem e retenção do dispositivo. Podem ser classificados em: a) AAM imediato: Monobloco, NAPA; b) AAM ajustáveis: Herbst, Klearway, EMA; c) AAM dinâmico: Aparelho Anti-Ronco (AAR-ITO) e Dispositivo Aperfeiçoado do Aparelho Anti-Ronco (DAAR-ITO) $3,14,21,23,25,26,32,33,35,36,37,60$.

Os aparelhos intrabucais são indicados para tratamento da SRVAS e da SAHOS leve, sendo considerados a primeira escolha para esses transtornos desde 1995 quando a American Sleep Disorders Association (agora, American Academy of Sleep Medicine) os reconheceu como alternativa eficaz à terapia com o CPAP.

É importante esclarecer que em casos de SAHOS grave, esses aparelhos podem ser utilizados, desde que outras modalidades terapêuticas tenham sido contra-indicadas ou recusadas. Nesses casos, devemos considerar que os dispositivos intrabucais representam parte do tratamento, que é multiprofissional e, portanto, necessitam de acompanhamento a longo prazo, além da cooperação do paciente no que se refere aos aspectos comportamentais, citados anteriormente ${ }^{36,37,60}$.

\section{1) Princípio de ação dos aparelhos intrabucais}

Uma vez elucidada a provável fisiopatologia da SAHOS, torna-se compreensível o princípio de ação dos aparelhos intrabucais, que promovem alterações nas estruturas anatômicas das vias aéreas superiores para manter a patência dessas vias durante a respiração noturna.

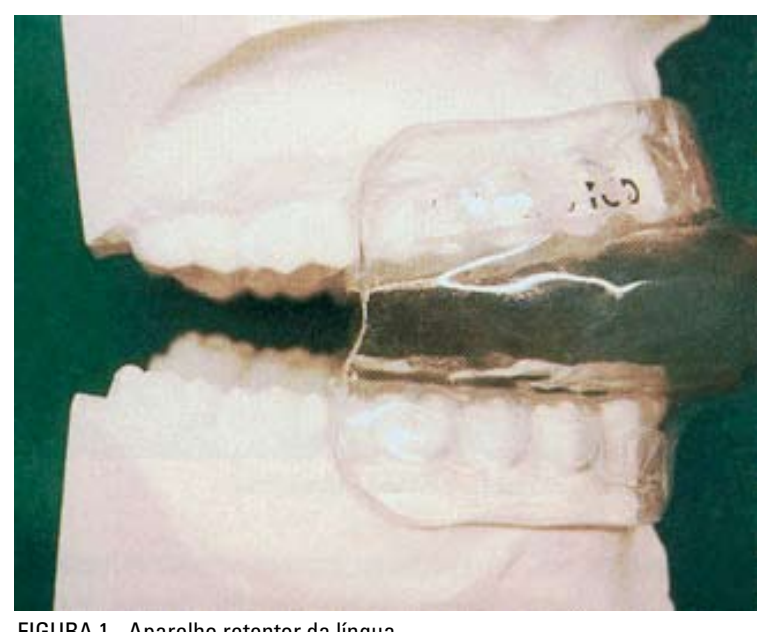

FIGURA 1 - Aparelho retentor da língua.

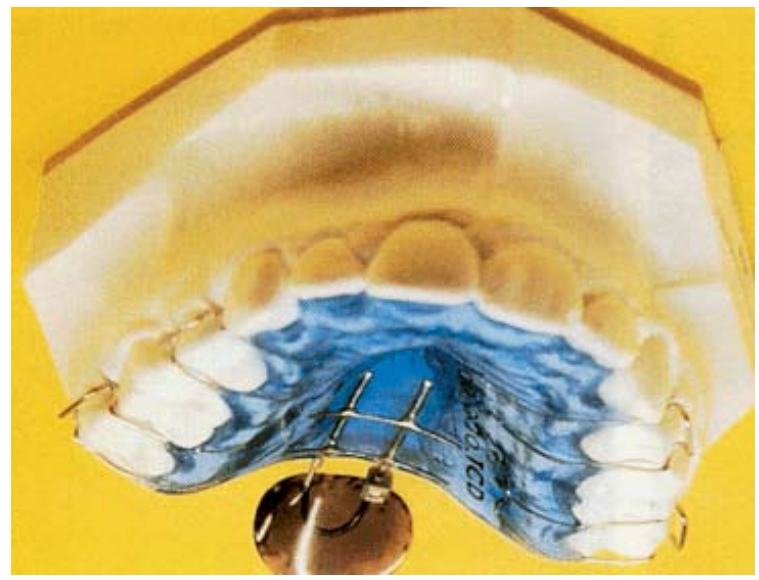

FIGURA 2 - Aparelho elevador do palato. 
As principais estruturas ósseas do complexo craniofacial que influenciam na dimensão das vias aéreas superiores são: a mandíbula e o osso hióide. A anteriorização mandibular, realizada pelos dispositivos de avanço mandibular, aumenta a atividade dos músculos genioglosso e pterigóideos laterais transmitindo tensões à musculatura supra e infra-hióidea, que, por conseqüência, irá proporcionar um posicionamento ântero-superior do osso hióide em relação à coluna cervical e ampliará as dimensões do conduto faríngeo. Essa nova situação anatômica é capaz de reduzir a vibração dos tecidos moles da orofaringe, que resulta no ronco, e assim, permitir a ventilação adequada durante o sono $^{4,8,25,31,36,37,45,70 \text {. }}$

Diversos estudos publicados na literatura, por análise cafalométrica, têm demonstrado, bidimensionalmente, que as alterações morfológicas, produzidas pelos aparelhos de avanço mandibular nas vias aéreas superiores e no posicionamento do hióide de pacientes com SAHOS, são capazes de promover aumento da porção posterior do espaço aéreo da faringe $3,8,9,31,45,52,60$.

Outros estudos, como os de Ryan ${ }^{61}$, Gale et al. ${ }^{24}$ e Kyung ${ }^{41}$ comprovaram, por meio de tomografia computadorizada na posição supina, em vigília, que o avanço mandibular, proporcionado pelos dispositivos intrabucais é capaz de ampliar tridimensionalmente o conduto faríngeo, mormente no plano lateral da região retropalatal e retroglossal, podendo este mecanismo ser o responsável pela redução significativa do IAH em pacientes sindrômicos.
3.2) A evolução dos Aparelhos Intrabucais de Avanço Mandibular (AAM)

A Odontologia vem progredindo no sentido de desenvolver um aparelho intrabucal ideal para tratar a SAHOS e a SRVAS. Assim, é notória a evolução que estas próteses para avanço mandibular sofreram nestas últimas décadas. Diversas modificações foram inseridas tanto no material utilizado para confecção, como no sistema de ancoragem e no mecanismo de ação desses aparelhos, com o intuito de aperfeiçoar o Monobloco para a utilização em pacientes adultos, tornando-os fisiológicos, mais confortáveis e, naturalmente, mantendo a efetividade ${ }^{37,38}$.

Em 2004, o autor e colaboradores ${ }^{36}$ publicaram revisão da literatura com os principais aparelhos de avanço mandibular quando enfocaram o mecanismo de ação e o sistema de retenção dos mesmos. Então, para situar o leitor quanto ao progresso desses dispositivos, faremos um resumo esquemático do que foi abordado:

\section{Aparelho Anti-Ronco® (AAR-ITO)}

Diferentemente dos dispositivos apresentados na literatura, o Aparelho Anti-Ronco® (AARITO) possui mecanismo de ação dinâmico que atua considerando a fisiologia neuromuscular do sistema estomatognático e o sono do paciente. Assim, permite que a mandíbula realize todos os movimentos fisiológicos e suas combinações, quando em posição durante o sono. Esse mecanismo é dependente da ação dos elásticos intermaxilares, os quais operam em sinergismo e sincronia com os
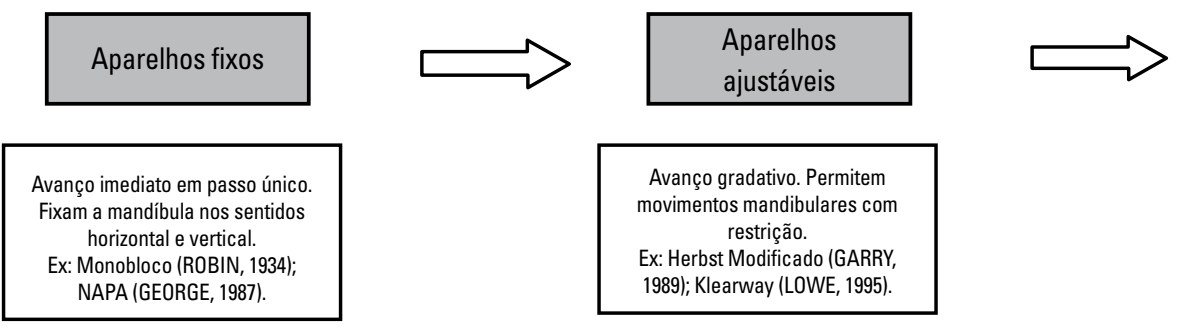

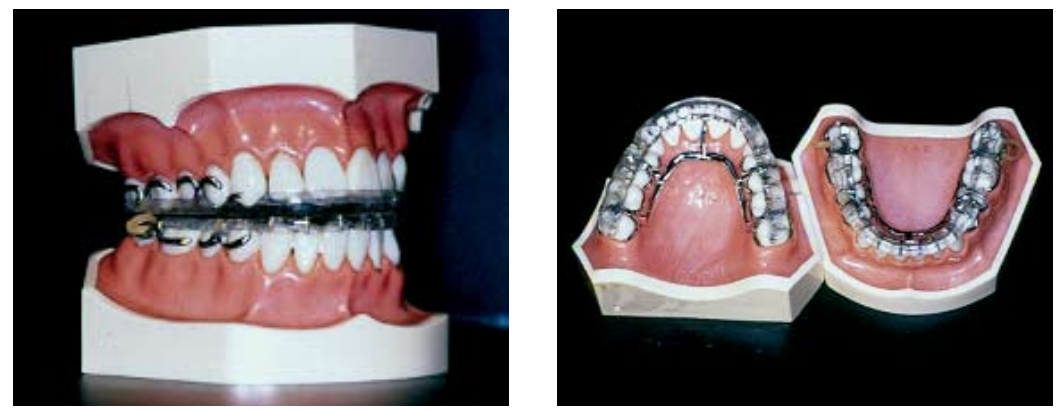

FIGURA 3 e 4 - AAR-ITO (com bases metálicas) montado em modelo padrão.

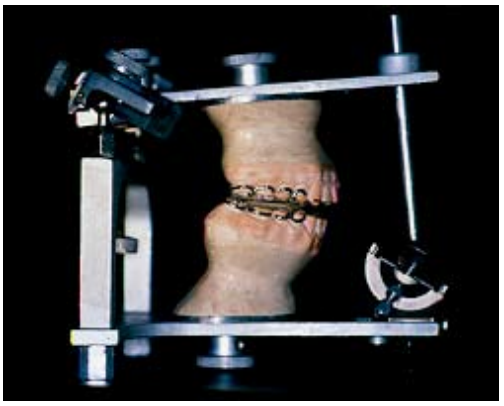

FIGURA 5 - AAR-ITO montado em articulador semiajustável. músculos da mastigação, principalmente com os músculos pterigóideos laterais de ambos os lados a partir da posição de isotonia muscular ${ }^{33,36,37}$.

O Aparelho Anti-Ronco® (AAR-ITO) é composto por duas bases metálicas (Cromo-Cobalto ou Níquel-Cromo) para Prótese Parcial Removível (PPR) modificadas, com opção para fios ortodônticos de aço inoxidável de secção transversal de $0,9 \mathrm{~mm}$, e duas placas miorrelaxantes (superior e inferior) de acrílico quimicamente ativado sobre cada estrutura metálica (Fig. 3 e 4). Essas placas miorrelaxantes são articuladas por intermédio de elásticos intermaxilares, em vetor Classe II (3/16" ou $1 / 8$ "), que são adaptados bilateralmente em grampos específicos localizados na região dos caninos superiores e dos molares inferiores ${ }^{33}$.

É confeccionado com auxílio de articulador semi-ajustável, a partir da posição de Máxima Intercuspidação Central (MIC) (Fig. 5) obtida por meio de ajuste oclusal ${ }^{33,35,36,37}$.

De acordo com a seqüência terápica proposta por Fairbanks e Fujita ${ }^{21}$, foi elaborado o protocolo clínico-odontológico para tratamento da SAHOS e SRVAS utilizando o Aparelho Anti-Ronco® (AAR-ITO). Por meio desse protocolo é possivel selecionar previamente o desenho mais adequado ao paciente considerando, dentre outros aspectos, a presença de todos os elementos dentários ou a perda parcial dos mesmos.

Conforme Ito et $\mathrm{al}^{37}$, as contra-indicações do Aparelho Anti-Ronco® são: 1) pacientes com de- sordens de natureza clínico-odontológica; 2) disfunção do sistema estomatognático em condição aguda ou subaguda e 3) pacientes não cooperadores e/ou desmotivados.

A seguir, descreveremos, o mecanismo dinâmico de ação do AAR-ITO relacionando-o com a arquitetura do sono:

\section{1) Anteriorização mandibular dinâmica}

a) Paciente em vigília: nesta fase há somente a ação dos elásticos intermaxilares em virtude da presença de tônus muscular que é uma das características do estado de consciência (Fig. 6);

b) Fase Hipnagógica: transição do estado de vigília para o estágio 1 do sono NREM: no final dessa fase, a ação dos elásticos intermaxilares promove um incremento no avanço mandibular devido ao relaxamento neuromuscular que se inicia (Fig. 7);

c) O relaxamento neuromuscular é proporcional ao aprofundamento dos estágios do sono NREM, ou seja, nos estágios 3 e 4 dessa fase do sono, esse relaxamento é maior, o que implica numa máxima anteriorização mandibular, podendo estar associado à abertura da cavidade bucal (Fig. 8);

\section{2) Posteriorização mandibular dinâmica}

Por reação à posição de anteriorização mandibular durante o sono, a mandíbula retornará para posição posterior a fim de atingir o repouso neuromuscular - isotonia (adjunta à posição de Relação Cêntrica). Antes de a mandíbula atingir a posição 
de descanso neuromuscular, o paciente poderá deglutir saliva, mantendo a lubrificação e a proteção dos tecidos orofaríngeos durante o sono (Fig. 9);

\section{3) Movimentos de lateralidade}

Os movimentos de lateralidade direita e esquerda completam a dinâmica do AAR-ITO permitindo que a mandíbula realize os movimentos fisiológicos, dinâmicos e suas combinações durante o sono (Fig. 10, 11).

Ao final da fase hipnopômpica (passagem do sono para a vigília), a dinâmica mandibular diminui e em seguida o paciente acorda.

\section{3) Discussão}

Vários dispositivos propostos na literatura são confeccionados a partir da posição de máxima intercuspidação habitual (MIH) do paciente, utilizando o método de tentativas (simulação clínica), para definir a quantidade de avanço mandibular. O fato de se ignorar o posicionamento neuromuscular do paciente durante o sono, poderá produzir diversos efeitos colaterais como alterações oclusais, dor orofacial e secura bucal 14,34,36,37,53,60,62,66,70.

Resultados iniciais, com controle polissonográfico, conseguidos com o AAR-ITO, indicam que não é condição sine qua non estabelecer previamente uma posição anteriorizada e fixa da mandíbula para se obter sucesso na terapia com os dispositivos intrabucais. Em termos fisiológicos, consideramos mais relevante posicionar a mandíbula neuromuscularmente, em isotonia, e promover a protrusão mandibular de forma dinâmica, a partir da posição de máxima intercuspidação central (MIC). Dentro desse contexto, é fundamental que, além de conceitos ortodônticos, sejam agregados aos dispositivos, conceitos multidisciplinares (Fisiologia, Oclusão e Prótese) para evitar a perpetuação dos efeitos colaterais ${ }^{36,38}$.

É prudente esclarecer que não há consenso determinando quanto (em milímetros) devemos avançar a mandíbula do paciente em vigília, para impedir a obstrução da orofaringe e, concomitan- temente, atuar em concordância com a fisiologia neuromuscular do paciente durante o sono, que, aliás, não está completamente esclarecida ${ }^{4,36,37}$.

Em adição, a complacência e a resiliência dos tecidos que compõem o sistema estomatognático, são fatores importantes para a manutenção da homeostasia desse sistema, principalmente na região das ATMs, que possuem mecanismo complexo e delicado de irrigação e inervação. É fundamental que os aparelhos intrabucais atuem de forma fisiológica, sem restringir os movimentos mandibulares, permitindo o descanso da musculatura, a deglutição de saliva e, conseqüentemente, a lubrificação e proteção dos dentes, tecidos orofaríngeos e trato gastrointestinal superior contra a microbiota patogênica e oscilações no $\mathrm{pH}$ esofágico durante o sono $^{35,36,37,50,66 \text {. }}$

De acordo com estudos realizados por Ito et al. ${ }^{36,37}$, as placas miorrelaxantes (superior e inferior) do AAR-ITO, devem ser confeccionadas limitadas ao espaço funcional livre (EFL) de $3 \mathrm{~mm}$ (média) para evitar o desconforto neuromuscular. Acréscimo na dimensão vertical de oclusão (DVO) aumenta as exigências de adaptação com conseqüências imprevisíveis para o equilíbrio oclusal e para a estabilidade do complexo côndilo-disco devido à possibilidade de conduzirem os côndilos mandibulares a posições excêntricas patológicas. Vale lembrar que alterações oclusais, desconforto miofacial e secura bucal (associada com despertar noturno para ingestão de água) são os efeitos colaterais mais freqüentes citados na literatu$\mathrm{ra}^{14,34,53,60,62,66,70}$

Em pacientes dolicocéfalos (com guia condilar profunda e ângulo mandibular aberto) a invasão do EFL e o conseqüente aumento da DVO, rotaciona a mandíbula no sentido horário causando constrição no diâmetro da orofaringe, prejudicando o benefício terapêutico conseguido com a anteriorização mandibular $8,36,37,49$.

\section{4) Tratamento farmacológico}

Uma grande variedade de medicamentos está 


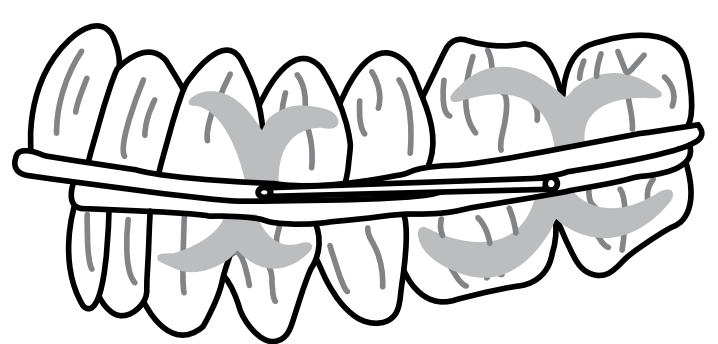

FIGURA 6 - Vista lateral - paciente em vigília.

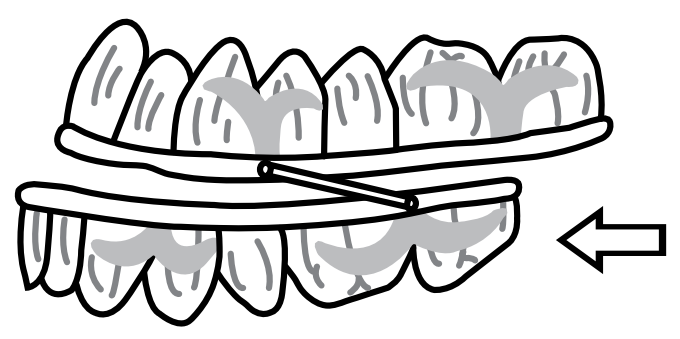

FIGURA 8 - Vista lateral - sono profundo.

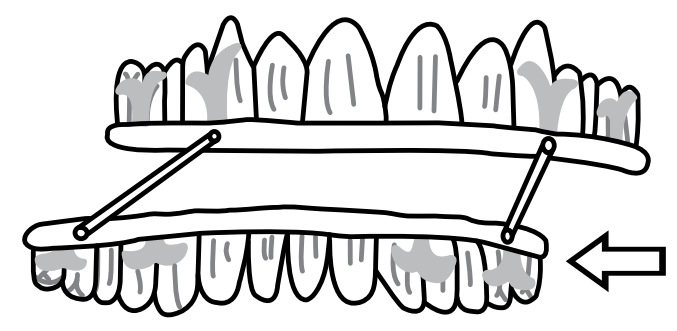

FIGURA 10 - Vista frontal - lateralidade direita durante o sono.

sendo testada para tratar a SAHOS, porém os resultados obtidos ainda não são conclusivos ${ }^{28,44}$.

\section{5) Tratamento cirúrgico}

Existem alternativas de tratamento baseadas na indicação de alguns procedimentos cirúrgicos. Os principais são:

5.1) Traqueostomia: foi o primeiro e mais eficaz método terapêutico utilizado para tratar a SAHOS grave $(\mathrm{IAH}>30)$, entretanto, está relacionado com complicações médicas (infecções, sangramento, irritação, dor) e problemas psicossociais. No momento a sua indicação fica restrita aos ca-

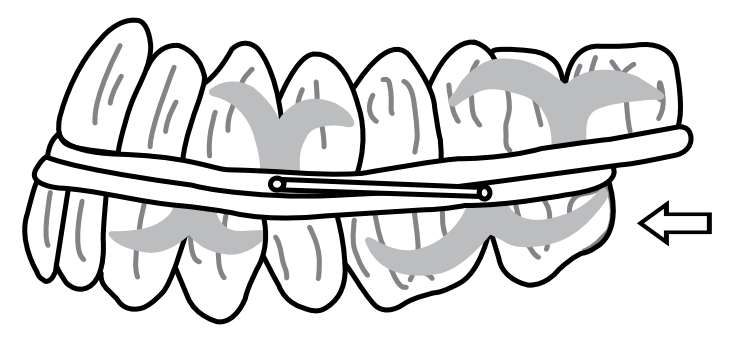

FIGURA 7 - Vista lateral - início do sono.

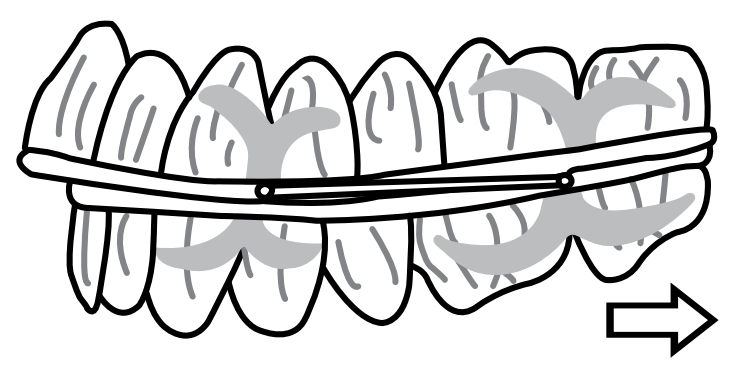

FIGURA 9 - Vista lateral quando o paciente engole durante o sono.

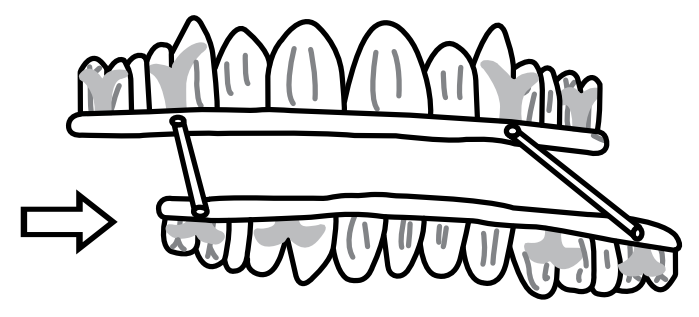

FIGURA 11 - Vista frontal - lateralidade esquerda durante o sono.

sos de intolerância ao CPAP e SAHOS grave associado à obesidade grau III com hipoxemia grave $\left(\mathrm{SatO}^{2}<50 \%\right)$ e repercussões cardiovasculares. De qualquer forma, é o único método que melhora quase que plenamente a apnéia do pacien$\mathrm{te}^{9,21,47}$.

5.2) Cirurgia nasal: diversas são as operações para a melhora da obstrução nasal. Metade dos pacientes que apresentam obstrução nasal não referem especificamente o sintoma, e sim, queixas indiretas, entre as quais pode-se incluir o ronco e a SAHOS. As afecções obstrutivas nasais mais freqüentes são rinite alérgica, desvio de septo, pó- 
lipos, hipertrofia de adenóide, alterações estruturais das conchas nasais e tumores. Sempre deve ser feita uma avaliação minuciosa da respiração nasal antes de se estabelecer qualquer tipo de tratamento para a SRVAS ou SAHOS, pois é uma causa comum $^{21,47,58}$.

\section{3) Uvulopalatoplastia (UPP) e Uvulopalatofa-} ringoplastia (UPFP): ambas são abordagens cirúrgicas, alternativas à traqueostomia, freqüentemente utilizadas para tratar a SRVAS e a SAHOS leve e moderada. A melhor indicação está nos indivíduos com IMC menor que $28 \mathrm{~kg} / \mathrm{m}^{2}$, boa respiração nasal, com tecido mucoso redundante e IAH entre 5 e 30 eventos/hora de sono. As técnicas visam diminuir o tecido mucoso abundante no palato fibroso, pilares amigdalianos, principalmente no posterior, e úvula. Podem ser utilizados diversos instrumentos cirúrgicos cortantes como: bisturi comum, radiofreqüência e laser, cada qual com suas vantagens e desvantagens. A escolha do tipo de anestesia, geral ou local, depende da técnica empregada e das condições clínicas e psíquicas do paciente. Os resultados da operação, respeitando as indicações corretas, são bons variando de $70 \%$ a $90 \%$ dos casos no primeiro ano de acompanhamento com alguma queda após. As complicações incluem voz anasalada, regurgitação nasal de líquidos, hemorragia, estenose nasofaríngea e secura da orofaringe, esta última notadamente quando não se reconstrói a úvula $5,20,21,30,42,44,67$.

5.4) Cirurgia ortognática: o objetivo desta cirurgia é avançar a mandíbula e/ou a maxila para se obter oclusão estável e perfil harmônico em pacientes retrognatas. Entretanto, tal avanço possibilita o aumento do espaço posterior das vias aéreas superiores devido ao deslocamento ventral da musculatura da faringe, do genioglosso e do osso hióide. Essa modalidade cirúrgica apresenta bons resultados para casos de SAHOS grave $(\mathrm{IAH}>30)$ em que o sítio obstrutivo se localiza na hipofaringe. O IMC elevado (maior que 38 $\mathrm{kg} / \mathrm{m}^{2}$ ) não é considerado critério de exclusão para essa técnica. $\mathrm{O}$ avanço maxilo-mandibular também pode ser utilizado como opção de resgate para o avanço do genioglosso e para a uvulopalatofaringoplastia, nos casos de SAHOS leve e moderada. A complicação mais comum desta cirurgia é a parestesia transitória do nervo alveolar inferior ${ }^{44,47,67,68}$.

5.5) Osteotomia mandibular com avanço do genioglosso: o objetivo dessa cirurgia é ampliar o espaço aéreo retrolingual. É indicada para os casos de obstrução retrolingual, retrognatia mandibular ( $<5 \mathrm{~mm}$ ) ou maxilomandibular, ambas com espaço aéreo posterior reduzido. Consiste na realização de janela óssea na região do mento, com subseqüente distensão da musculatura do assoalho da boca e do genioglosso em cerca de 10 a $14 \mathrm{~mm}$. A fixação do fragmento ósseo é rígida e na sua posição original ${ }^{30}$.

5.6) Canalização da língua: Consiste na abertura em forma de U no dorso lingual e remoção de tecido, por intermédio de raios laser, com o objetivo de aumentar a passagem do ar nos pacientes que apresentam a língua excessivamente volumosa em sua porção póstero-superior. Tem o inconveniente de sangrar excessivamente e, as vezes, requerer traqueostomia de urgência. Da mesma forma que a técnica anterior, não se conhecem os resultados a longo $\operatorname{prazo}^{21,58}$.

\section{6) Terapias combinadas}

A associação entre CPAP e os aparelhos intrabucais tem sido relatada na literatura, assim como a utilização do CPAP antecedendo cirurgias em pacientes obesos. Os dispositivos intrabucais podem ser utilizados temporariamente para substituir o CPAP em situações onde não haja fornecimento de energia elétrica. A UPFP pode ser combinada com cirurgias nasais (exérese de pólipos, septoplastia) ou associada à 
amigdalectomia das palatinas, quando estas forem volumosas. As medidas comportamentais são utilizadas paralelamente com o CPAP e com os aparelhos intrabucais, devendo ser mantidas a longo prazo. As terapias acima citadas podem ser utilizadas conjuntamente ou seqüencialmente com o objetivo de melhorar a eficácia terapêutica ${ }^{12,14,19,51,60}$.

\section{EFICÁCIA DO TRATAMENTO}

Para verificar a efetividade do tratamento proposto, é necessário que os profissionais dedicados ao tratamento dos transtornos do sono sejam criteriosos ao avaliar os parâmetros clínicos e laboratoriais (Tab. 2), que são fundamentais para indicar se houve sucesso com a terapia proposta ou se haverá necessidade de se instituir outro tipo de tratamento ${ }^{13}$.

A terapia com os aparelhos intrabucais requer controle polissonográfico e reavaliações, de ordem médica e odontológica, periódicas.

O cirurgião-dentista deverá estar apto para solucionar prontamente as possiveis intercorrências clínicas (irritação da mucosa bucal e lingual, dificuldade de retenção do aparelho na cavidade bucal durante o sono, fratura de restaurações, e desconforto dental) e efeitos colaterais já citados ${ }^{36,37,70}$.

\section{CONCLUSÕES}

Em conformidade com a literatura revisada, podemos concluir que:

1) Devido à alta incidência e prevalência dos transtornos citados e de suas sérias conseqüências que atingem direta e indiretamente diversos setores sócio-econômicos, suscitando custos significativos tanto para o Estado como para as empresas, os transtornos do sono surgem, nestas últimas três décadas, como uma das perturbações mais comuns das nossas funções diuturnas. Além disso, identificam-se correlações suas com doenças sistêmicas insuspeitadas anteriormente. A obesidade, hipertensão arterial sistêmica e os transtornos do sono constituem uma tríade epidêmica do fim do milênio, que somadas às doenças infecciosas emergentes e reemergentes, formam um quarteto que engloba grande parte dos problemas de saúde pública.

2) A decisão, dentre as diversas estratégias de tratamento para a SRVAS e para a SAHOS apresentadas, irá depender do diagnóstico diferencial de um médico especialista em Medicina do Sono, associado ao laudo do exame de polissonografia para se estabelecer um plano de tratamento adequado ao paciente;

3) A integração entre a Medicina e a Odontologia é fundamental para o tratamento dos transtornos do sono em questão, propiciando estabelecer, mediante solicitação médica, um tratamento conservador, no qual a intervenção com os aparelhos intrabucais é considerada opção eficaz. Após a solicitação médica, cabe ao odontólogo, mediante protocolo clínico, executar o tratamento e acompanhar clínica e polissonograficamente a evolução dos casos até que o controle satisfatório seja estabelecido.

4) É importante que o odontólogo, enquanto membro de equipe multiprofissional que está voltada para o tratamento dos transtornos do sono (SAHOS, SRVAS, Bruxismo), adquira conhecimentos sobre diagnóstico diferencial, indicações terapêuticas - princípios de ação que devem nortear a indicação do aparelho intrabucal, estratégias de tratamento e a fisiopatologia

\footnotetext{
Tabela 2 - Parâmetros para avaliação da eficácia do tratamento.

Parâmetros laboratoriais

Redução significativa ou normalização do Índice de Apnéia e Hipopnéia (IAH)

Redução significativa dos microdespertares

Melhora da dessaturação de oxihemoglobina

Ausência de arritmia cardíaca

Fonte: Asda (1995); Cartwright (2001)

\section{Parâmetros clínicos}

Redução significativa ou eliminação do ronco

Melhora no grau de hipersonolência diurna

Satisfação do paciente quanto ao tratamento proposto
} 
desses transtornos. Portanto, é necessário que os profissionais da Odontologia se mantenham atualizados e possuam currículo em Medicina do Sono para que possam atuar de forma segura no tratamento desses transtornos, já que esses conhecimentos ainda não são do domínio de sua formação acadêmica.

5) A utilização dos dispositivos intrabucais no tratamento da SRVAS e da SAHOS tem merecido a atenção de diversas especialidades da área de saúde devido à sua aceitabilidade e efetividade para os casos de SRVAS e SAHOS leve. A dificuldade de receptividade para outras opções terapêuticas torna o uso dos aparelhos intrabucais uma alternativa de alta validade porque são conservadores, reversíveis e apresentam o melhor custo-benefício.

Enviado em: Maio de 2004 Revisado e aceito: Janeiro de 2005

\title{
Obstructive Sleep Apnea and Hypopnea Syndrome (OSAHS) and snoring: treat- ment strategies with focus in Mandibular Advancement Device ${ }^{\circledR}$ (MAD-ITO)
}

\begin{abstract}
The authors present a literature review of the surgical and non-surgical treatment alternatives to the Obstructive Sleep Apnea and Hypopnea Syndrome (OSAHS) and snoring with focus in the mechanism of action and progress of mandibular advancement devices mainly in the dynamic action mechanism of the Mandibular Advancement Device® (MAD-ITO).
\end{abstract}

Key words: Mandibular Advancement Device ${ }^{\circ}$ (MAD-ITO). Dynamic action mechanism. Obstructive Sleep Apnea and Hypopnea Syndrome (OSAHS). Snoring.

\section{REFERÊNCIAS}

1. AMERICAN SLEEP DISORDERS ASSOCIATION (ASDA). Practice parameters for the treatment of snoring and obstructive apnea with oral appliances. Sleep, New York, v.18, no.6 p. 511-513, 1995.

2. ASERINSKY, E.; KLEITMAN, N. Regularly occurring periods of ocular mobility and concomitant phenomena during sleep. Science, Washington, D. C. v. 118, p. 273-274, 1953.

3. ATHANASIOU, A. E.; PAPADOPOULOS, M. A.; MAZAHERI, M.; LAGOUDAKIS, M. Cephalometric evaluation of pharynx, soft palate, adenoid tissue, tongue and hyoid bone following the use of a mandibular repositioning appliance in obstructive sleep apnea patients. Int J Adult Orthodon Orthognath Surg. Chicago, v. 9, no. 4, p. 273-283, 1994.

4. AYAPPA, I.; RAPOPORT, D. M. The upper airway in sleep: physiology of the pharynx. Sleep Medicine Reviews, London, v. 7, no. 1, p. 9-33, 2003.

5. BACK, G. B.; NADIG, S.; UPPAL, S.; COATESWORTH, A. P. Why do we have a uvula? Literature review and a new theory. Clin Otolaryngol, Oxford, v. 29, p. 689-693, 2004.

6. BATTAGEL, J. M. Obstructive sleep apnea: fact not fiction. Br J Orthod, London, v. 23, no. 4, p. 315-324, 1996.
7. BERGER, R. J. Tonus of extrinsic laryngeal muscles during sleep and dreaming. Science, Washington, D.C., v. 134, p. 840, 1961.

8. BERNHOLD, M.; BONDEMARK, L. A. A magnetic appliance for treatment of snoring patients with and without obstructive seep apnea. Am J Orthod Dentofacial Orthop, St. Louis, v. 113, p. $144-155,1998$.

9. BONHAM, P. E.; CURRIER, G. F.; ORR, W. C.; OTHMAN, J. NANDA, R. S. The effect of a modified functional appliance on obstructive sleep apnea. Am J Orthod Dentofacial Orthop St. Louis, v. 94, p. 384-392, 1988.

10. BRAVER, H. M.; BLOCK, A. J.; PERRI, M. G. Treatment for snoring: combined weight loss, sleeping on side and nasal spray. Chest, Chicago, v. 107, p. 1283-1288, 1995.

11. CARTWRIGHT, R.; SAMELSON, C. F. The effects of a nonsurgical treatment for obstructive sleep apnea - the tongue-retaining device. JAMA, Chicago, v. 248, no. 6, p. 705-709, 1982.

12. CARTWRIGHT, R.; RISTANOVIC, R.; DIAZ, F.; CALDARELLI, D.; ALDER, G. A comparative study of treatments for positional sleep apnea. Sleep, New York, v. 14, p. 546-552, 1991.

13. CARTWRIGHT, R. What's new in oral appliance for snoring and sleep apnea: an update. Sleep Medicine Reviews, London, v. 5, no. 1, p. 25-32, 2001.

14. CLARK, G. T. Mandibular advancement devices and sleep disordered breathing. Sleep Medicine Reviews, London, v. 2, no. 3 , p. 163-174, 1998. 
15. COY, T. V: DIMSDALE, J. E.: ANCOLI-ISRAEL, S: CLAUSEN, J. $\mathrm{L}$. The role of sleep disordered breathing in essential hypertension. Chest, Chicago, v. 108, p. 890-895, 1996.

16. CROCKER, B. D.; OLSON, L. G.; SAUNDERS, N. A.; HENSLEY, M. J.; MCKEON, J. L.; ALLEN, K. M.; GYULAY, S. G. Estimation of the probability of disturbed breathing during sleep before a sleep study. Am Rev Respir Dis, Baltimore, v. 142, p. 14-18, 1990.

17. DAWSON, P. E. Avaliação, diagnóstico e tratamento dos problemas oclusais. São Paulo: Artes Médicas, 1993. p. 63-79, 93-99

18. DIAGNOSTIC Classification Steering Committee. The International Classification of Sleep Disorders, revised: diagnostic and coding manual. Rochester: American Sleep Disorders Association, 1997.

19. DOUGLAS, N. J.; POLLO, O. Pathogenesis of obstructive sleep apnoea/hypopnoea syndrome. Lancet, London, v. 344, p. 653-655, 1994.

20. ENGSTRÖM, M. L. W.; WILHELMSSON, B.; TEGELBERG, A.; DIMENÄS, E.; RINGQVIST, I. Quality of life assessment of treatment with dental appliance or UPPP in patients with mild to moderate obstructive sleep apnoea. A prospective randomized 1-year follow-up study. J Sleep Res, Oxford, v. 9, p.303-308

21. FAIRBANKS, D. N. F.; FUJITA, S. Snoring and obstructive sleep apnea. 2nd ed. New York: Raven Press, 1995.

22. FLETCHER, E. C.; LUCKET, R. A. The effect of positive reinforcement an hourly compliance in nasal continuous positive airway pressure users in obstructive sleep apnea. Am Rev Respir Dis, Baltimore, v. 143, p. 936-941, 1991.

23. HENKE, K. G.; FRANTZ, D. E.; KUNA, S. T. An oral elastic mandibular advancement device for obstructive sleep apnea. Am J Respir Crit Care Med, New York, v. 161, p. 420-425, 2000.

24. GALE, D. J.; SAWYER, R. H.; WOODCOCK, A.; STONE, P.; THOMPSON, R.; O'BRIEN, K. Do oral appliances enlarger the airway in patients with obstructive sleep apnea? A prospective computerized tomographic study. Eur J Orthod, London, v. 22, p. 159-168, 2000.

25. GARRY, J. F. Obstructive sleep apnea syndrome and the GarryPrior Modified Herbst Appliance. Space Maintainers Newsletter, [S.I.], v. 8, p. 2, 1989.

26. GEORGE, P. A modified functional appliance for treatment of obstructive sleep apnea. J Clin Orthod, Boulder, IV. 16, p. 171-175, 1987.

27. GUILLEMINAULT, C. Obstructive sleep apnea: The clinical syndrome and historical perspective. Med Clin North Am, Philadelphia, v. 69, no. 6, p. 1187-1203, 1985.

28. HANZEL, D. A.; PROEA, N. G.; HUDGEL, D. W. Response of obstructive sleep apnea to fluoxetine and protriptyline. Chest, Chicago, v. 100, no. 2, p. 416-421, 1991.

29. HE, J.; KRYGER, M. H.; ZORICK, F. J.; CONWAY, W.; ROTH, T. Mortality and apnea index in obstructive sleep apnea: experience in 385 male patients. Chest, Chicago, v. 94, p. 9-14, 1988.

30. HENDLER, B. H.; COSTELLO, B. J.; SILVERSTEIN, K.; YEN, D.; GOLDBERG, A. A protocol for uvulopalatopharyngoplasty, mortised genioplasty, and maxillomandibular advancement in patients with obstructive sleep apnea: an anlysis of 40 cases. J Oral Maxillofac Surg, Philadelphia, v. 59, p. 892-897, 2001.

31. HOEKEMA, A.; HOVINGA, B.; STEGENGA, B.; DE BONT, L. G. M. Craniofacial morphology and obstructive sleep apnoea: a cephalometric analysis. J Oral Rehabil, Oxford, v. 30, p. 690-696, 2003.

32. INSTITUTO NACIONAL DA PROPRIEDADE INDUSTRIAL (INPI). Modelo de utilidade: 8500840-0. Rio de Janeiro, 2005. 8500840-0.

33. INSTITUTO NACIONAL DA PROPRIEDADE INDUSTRIAL (INPI). Patente de Invenção (PI) - $\mathrm{n}^{\circ} 00016357$. Rio de Janeiro, 2000.

34. ITO, F. A. Anatomia relacionada à síndrome da apnéia e hipopnéia obstrutiva do sono, ao ronco e ao Aparelho AntiRonco®. 2002. Trabalho de Conclusão de Curso (Especialização)- Faculdade de Odontologia, Universidade de São Paulo, São Paulo, 2002.
35. ITO, F. A.; ITO, R. T.; MORAES, N. M.; BEZERRA, M. L. S. Mecanismo de ação dinâmico do Aparelho Anti-Ronco ${ }^{\circledR}$ (AAR). CONGRESSO BRASILEIRO DE SONO, 9., 2003. [S. I.], Anais... [S.I.: s.n.], 2003 p. 87-88

36. ITO, F. A.; ITO, R. T.; MORAES, N. M.; SAKIMA, T.; BEZERRA, M.

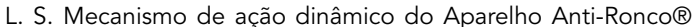
(AAR): Relato de um caso clínico. R Clin Ortodon Dental Press, Maringá, v. 3, no. 1, p. 41-50, 2004.

37. ITO, R. T.; ITO, F. A.; MELO, A. C.; BRONZI, E. S.; YOSHIDA, A H.; YOSHIDA, N.; SAKIMA, T. Aparelho anti-ronco: um enfoque multidisciplinar. R Dental Press Ortodon Ortop Facial, Maringá, v. 5, n. 2, p. 48-53, 2000.

38. ITO, F. A.; MORAES, N. M. Aparelho Anti-Ronco® (AAR-ITO) no tratamento da síndrome da apnéia e hipopnéia obstrutiva do sono. ENCONTRO CIENTÍFICO DE ODONTOLOGIA DO ESTADO DO RIO DE JANEIRO, 2; FÓRUM ODONTOLOGIA PARA PACIENTES COM NECESSIDADES ESPECIAIS, 5., 2004, [S. I.]. Resumo em anais... [S.I. : s.n.], 2004.

39. JUNG, R.; KUHLO, W. Neurophysiological studies of abnormal night sleep and pickwickian syndrome. Prog Brain Res, Amsterdam, v. 18, p. 140-159, 1965

40. KUNA, S. T.; SANTÁMBROGIO, G. Pathophysiology of upper airway closure during sleep. JAMA, Chicago, v. 266, no. 10, p. $1384-1389,1991$

41. KYUNG, S. H.; PARK, Y. C.; PAE, Y. K. Obstructive sleep apnea with the oral appliance. Experience pharyngeal size and shape changes in three dimensions. Angle Orthod, Appleton, v. 75, p. 15-22, 2004.

42. LARSSON, L. H.; CARLSON-NORDLANDER, B.; SVANBORG, E. Four-year follow up after uvulopalatopharyngoplasty in 50 unselected patients with obstructive sleep apnea syndrome. Laryngoscope, St. Louis, v. 104, p.1362-1369, 1994.

43. LAVIE, P.; BERGER, I.; YOFFE, N.; RUBIN, A.; CHILLAG, N. Longterm morbidity and mortality of SAS patients. J Sleep Res, Oxford, v. 3, p. 131, 1992. Supplement, 1.

44. LÉVY, P. et al. Management of simple snoring, upper airway resistance syndrome and moderate sleep apnea syndrome. Sleep, New York, v. 19, no. 9, p. 101-110, 1996.

45. LOWE, A. A.; ONO, T.; FERGUSON, K. A.; PAE, E. K.; RYAN, F.; FLEETHAM, J. A. Cephalometric comparisons of craniofacial and upper airway structure by skeletal subtype and gender in patients with obstructive sleep apnea. Am J Orthod Dentofacial Orthop, St. Louis, v. 110, no. 6, 1996.

46. MANSON, J. E.; WILLET, W. C.; STAMPFER, M. J.; COLDITZ, J. A.; HUNTER, D. J.; HANKINSON, S. E. Body weight and mortality among women. N Engl J Med, Boston, v. 333, p. 677-685, 1995.

47. MARTINEZ, D. Prática da medicina do sono. São Paulo: Fundo Editorial BYK, 1999. p. 16-45.

48. MEIER-EWERT, K.; SCHAFER, H.; KLOSS, W. Treatment of sleep apnea by a mandibular protracting device. 7th EUROPEAN CONGRESS ON SLEEP RESEARCH, 7., 1984, [S. I.], Abstract Book... [S. I.: s.n.], 1984. p. 217.

49. MEURICE, J.; MARC, I.; CARRIER, G.; SERIES, F. Effects of mouth opening on upper airway collapsibility in normal sleeping subjects. Am J Respir Crit Care Med, New York, v. 153, p. 255259, 1996.

50. MIYAWAKI, S.; TANIMOTO, Y.; ARAKI, Y.; KATAYAMA, A.; IMAI, M.; TAKANO-YAMAMOTO, T. Relationships among nocturnal jaw muscle activities, decreased esophageal $\mathrm{pH}$, and sleep positions. Am J Orthod Dentofacial Orthop, St. Louis, v. 126, p. $615-619,2004$

51. ONO, T., O.; LOWE, A. A.; FERGUSON, K. A.; PAE, E. K.; FLEETHAM, J. A. The effect of tongue retaining device on awake genioglossus muscle activity patients with obstructive sleep apnea. Am J Orthod Dentofacial Orthop, St. Louis, v. 110, no. 1, 1996.

52. PAE, E.; LOWE, A. A.; SASAKI, K.; PRICE, C.; TSUCHYIA, M.; FLEETHAM, J. A. A cephalometric and electromyographic study of upper airway structures in the upright and supine position. Am J Orthod Dentofacial Orthop, St. Louis, v. 106, p. 52-59, 1994. 
53. PANTIN, C. C. HILLMAN, D. R. TENNANT, M. Dental side effects of an oral device to treat snoring and obstructive sleep apnea. Sleep, New York, v. 22, no. 2, p. 237-240, 1999.

54. PASKOW, $\mathrm{H}$; PASKOW, S. Dentistry role in treatment sleep apnea and snoring. N Engl J Med, Boston, v. 88, p. 815-817, 1991.

55. PEISER, J.; LAVIE, P.; OVNAT, A.; CHARUZI, I. Sleep apnea syndrome in the morbidly obese as an indication for weight reduction surgery. Ann Surg, Philadelphia, v. 100, p.112, 1984

56. PHILLIPSON, E. A. Sleep apnea: a major public health problem. N Engl J Med, Boston, v. 382, p. 1271-1273, 1993.

57. POYARES, D.; ALOE, F.; RIZZO, G. N. V. et al. $1^{\circ}$ Consenso Brasileiro de Insônia. Hypnos, [S. I.], ano 4, 2003. Suplemento 2

58. REIMÃO, R. Sono estudo abrangente. São Paulo: Atheneu, 1996.

59. REMMERS, J. E.; STERLING, J. A.; THORARINSSON, B.; KUNA, S. T. Nasal airway positive pressure in patients with occlusive sleep apnea: methods and feasibility. Am Rev Respir Dis, Baltimore, v. 130, no. 6, p. 1152-1155, 1984

60. ROSE, E. C.; BARTHLEM, G. M.; STAATS, R.; JONAS, I. E. Therapeutic efficacy of an oral appliance in the treatment of obstructive sleep apnea: a 2-year follow up. Am J Orthod Dentofacial Orthop, St. Louis, v. 121, no. 3, p. 273-279, 2002

61. RYAN, C. F.; LOWE, L. L.; PEAT, D.; FLEETHAM, J. A.; LOWE, A. A. mandibular advancement oral appliance therapy for obstructive sleep apnea: effect on awake caliber of velopharynx. Thorax, London, v. 54, p. 972-977, 1999

62. SCHMIDT-NOWARA, W.; LOWE, A.; WIEGAND, L.; CARTWRIGHT, R.; PEREZ-GUERRA, F.; MENN, S. Oral appliances for the treatment of snoring and obstructive sleep apnea: a review. Sleep, New York, v. 18, no. 6, p. 501-510, 1995

63. SCRIMA, L.; BROUDY, M.; MAY, K. N. Increased severity of obstructive sleep apnea after bedtime alcohol ingestion: diagnostic potential and proposed mechanism of action. Sleep, New York v. 5, p. 318-322, 1982.

64. STRADLING, J. R. Sleep apnea and systemic hypertension. Thorax, London, v. 44, p. 984-989, 1989

65. SULLIVAN, C. E.; ISSA, F. G.; BERTHON, J. M. Reversal of obstructive sleep apnea by continuous positive airway pressure applied through the nares. Lancet, London, v. 1, p. $862-865$ 1981

66. THIE, N. M. R.; KATO, T.; BADER, G.; MONTPLAISIR, J. Y.; LA VIGNE, G. J. The significance of saliva during sleep and the relevance of oromotor movements. Sleep Medicine Reviews London, v. 6, no. 3, p. 213-227, 2002

67. TUFIK, S.; SAMPAIO, P. L.; WECKX, L. L. M. et al. Congresso brasileiro em ronco e apnéia do sono, Hypnos, 1., 2001. [S. I. ] Anais.... [S.I.: s. n.], 2001., suplemento 1. 2001.

68. VEIS, R. W. Snoring and sleep apnea from dental perspective. J Calif Dent Assoc, San Francisco v. 26, no. 8, p. 557-565, 1998

69. WALDHORN, R. E. Sleep apnea syndrome. Am Fam Physician Kansas City, v. 32, no. 3, p. 149-166, 1985.

70. WARUNEK, S. P. Oral appliance therapy in sleep apnea syndromes: a review. Semin Orthod, Philadelphia, v. 10, no.1, Mar. p. 73-89, 2004

71. WEIGAND, L.; ZWILICH, C. W. Obstructive sleep apnea In: BONE, R. C. R. Disease month. St. Louis: Mosby Year Book, 1994. p. 199-252.

72. YILDIRIM, N.; FITZPATRIC, M. F.; WHYTE, K. F.; JALLEH, R. WIGHTMAN, A. J. A.; DOUGLAS, N. J. The effect of posture on upper airway dimensions in normal subjects and in patients with the sleep apnea/hypopnea syndrome. Am Rev Respir Dis Baltimore, v. 144, p. 585-847, 1991

73. YOUNG, T.; PALTA, M.; DEMPSEY, J.; SKATRUD, J.; WEBER, S.; BADR, S. The occurrence of sleep-disordered breathing among middle-aged adults. N Engl J Med, Boston, v. 328, p. 1230 1235, 1993.

\section{Endereço para correspondência}

Centro Odontológico de Pesquisas Clínicas

Dr. Roberto Ito \& Dr. Fausto Ito Ltda.

Rua Visconde de Pirajá 463 SI. 210

lpanema - Rio de Janeiro/RJ

CEP 22.410003

e-mail: faustoito@gmail.com 
O arquivo disponível sofreu correções conforme ERRATA publicada no Volume 10 Número 6 da revista. 